\section{P88 Is it Just a Measurement? Collecting, Tracking, and Reporting BMI in Head Start}

M. Elizabeth Miller, PhD, LDN, RDN, FAND, mille736@miamioh.edu, Miami University, 420 S Oak St, Oxford, OH, 45056; Stephanie Nicely, EdD, RN, Miami University; Dana Shefet, East Carolina University; Virginia C. Stage, PhD, RDN, East Carolina University

Background: Head Start (HS) is required to collect height and weight data of preschoolers each year and report aggregate rates of BMI. However, limited research has been conducted to understand this process.

Objective: This study explored the processes HS programs in Ohio and North Carolina use to measure, track, and report children's weight status.

Study Design, Setting, Participants: This qualitative study utilized a phenomenological approach. Twentynine semi-structured interviews were conducted with Head Start Health and Nutrition Managers across Ohio (n $=14)$ and North Carolina $(n=15)$. Interviews were conducted until saturation was achieved.

Measurable Outcome/Analysis: Interviews were recorded and transcribed verbatim. Transcripts were independently reviewed and significant statements were identified, describing what participants experienced. Patterns of meaning across interviews were assigned codes and described. Codes were compared, agreed upon, and organized into themes.

Results: Analysis yielded 5 themes: perceptions of BMI, measurement processes, reporting/tracking, training and resources, and additional support. Managers described a variety of concerns with the usefulness of BMI. Differences were identified in the staff responsible for measurements, as well as their expertise and training. Equipment used for measuring height and weight also varied across and within programs. Most managers reported utilizing computer programs where height and weight were entered, BMI calculated, and weight status classifications generated for reporting to parents and on the Program Information Report. Managers acknowledged inconsistencies in the persons responsible for data collection, training of collectors, and variability in the data collection process may impact accuracy of reporting. Furthermore, no consistent process was identified for the reporting and referral of atrisk children.

Conclusion: Training on collection of height and weight measurements is needed, as most programs described limited availability of training opportunities, resources, and time to implement internal training on growth and assessment. Additionally, clarification and standardization of the process for tracking, reporting and referring at-risk children is warranted.

Funding: College of Education, Health and Society at Miami University (Ohio) and a College of Allied Health Sciences Thesis Award at East Carolina University.

\section{P89 "Karmically Awesome Pairings": A Qualitative Analysis of SNEB Mentorship Program Participant Feedback Surveys}

April Williams, MS,LDN, CNS, unamela004@gmail.com, Virginia Commonwealth University, 830 E Main St, Richmond, VA, 23219; Kritika Gupta, MS, University of Mississippi; Graham E. Bastian, RDN, Rutgers, The State University of New Jersey; Het Desai-Shah, BS, Rutgers, The State University of New Jersey; Zubaida Qamar, PhD, RD, San Francisco State University; Megan Patton-Lopez, PhD, $R D N$, Western Oregon University

Background: Mentorship programs in professional organizations can benefit both mentors and mentees by providing support, community, networking, and knowledgesharing. The current virtual environment provides a novel opportunity to expand the dimensions of the mentorship program for years ahead.

Objective: To conduct a secondary qualitative analysis of survey responses from former SNEB Mentorship Program participants as part of formative research for the development of future programming.

Study Design, Setting, Participants: Data were obtained from satisfaction surveys completed by mentors and mentees who participated in the mentorship program at SNEB Annual Conferences between 2012-14 and 2016-17.

Measurable Outcome/Analysis: Two independent coders performed an inductive thematic analysis of responses to open-ended survey questions to determine program characteristics associated with participants' satisfaction to inform program expansion. Findings were reviewed by 2 additional researchers and final themes were agreed upon by all the researchers.

Results: Themes touting program benefits emerged from an analysis of survey responses $(\mathrm{n}=112)$ included "flexibility," "networking", "support," "welcoming personalities of mentors," "impact of the program," and "appropriate matching of mentors and mentees." "Structure" and "communication" were themes that emerged from coding both mentor and mentee responses $(n=85)$ to inquiry about program improvements. Mentees desired longer mentorship periods, whereas mentors proposed having a designated session to meet and greet the mentees and having substitute mentors available.

Conclusion: The program was well-received by participants. Both mentors and mentees expressed varying degrees of interest in expanding the mentorship program beyond the conference. Future mentorship programs should incorporate elements of flexibility but structure in communication between the 2 parties to have a more meaningful impact on the mentor-mentee relationship.

Funding: None. 